Focussed on "Crack Paths"

\title{
A model of energy dissipation at fatigue crack tip in metals
}

\author{
Oleg Plekhov, Aleksei Vshivkov, Anastasia Iziumova \\ Institute of Continuous Media Mechanics Russian Academy of Sciences Ural Branch \\ poa@icmm.ru,vshivkov.a@icmm.ru,fedorova@icmm.ru
}

Balasubramaniam Venkatraman

Indira Gandhi Centre for Atomic Research, Tamil Nadu, Kaplakkam, India

bvenkat@igcar.gov.in

\begin{abstract}
Experimental and numerical studies investigating fatigue crack growth were conducted on flat samples made of stainless steel AISE 304 and titanium alloy Grade 2. The heat flux from the crack tip caused by plastic deformation localization was measured using the contact heat flux sensor previously developed by the authors. This provides a possibility to find a correlation between energy dissipation and crack propagation rate under fatigue uniaxial loading with constant stress intensity factor and under biaxial loading with constant stress amplitude. The experiments with constant stress intensity factor have shown a decrease in energy dissipation at constant crack rate and stress ratio $\mathrm{R}=\sigma_{\min } / \sigma_{\max }=-1(\mathrm{R}=0)$. A theoretical analysis of the stress field at the fatigue crack tip has been carried out to explain this phenomenon. Based on the obtained results, the heat flux from the crack tip is represented as the sum of two functions describing energy dissipation in monotonic and reversible plastic zones separately. It has been shown that dissipation in a reversible plastic zone is a function of the applied stress amplitude only. This causes a decrease of energy dissipation at constant stress intensity factor. The proposed phenomenology was successfully verified by testing both materials under biaxial loading.
\end{abstract}

OPEN ACCESS

Citation: Plekhov, O., Vshivkov, A., Iziumova, A., Venkatraman, B., A model of energy dissipation at fatigue crack tip in metals, Frattura ed Integrità Strutturale, 48 (2019) 451-458.

Received: 28.11 .2018

Accepted: 04.02 .2019

Published: 01.04.2019

Copyright: (C) 2019 This is an open access article under the terms of the CC-BY 4.0, which permits unrestricted use, distribution, and reproduction in any medium, provided the original author and source are credited.

KEYWORDS. Fatigue crack; Dissipated energy; Plastic deformation zone.

\section{INTRODUCTION}

7 he kinetics of fatigue crack growth over a wide range of crack propagation rates can be described using a correlation with stress intensity factor (Paris`s law). This correlation is the result of approximation of a large amount of experimental data and it does not explain the physical nature of this process. 
Many authors proposed other correlations between the fatigue crack rate and different mechanical-structural parameters. For instance, the J-integral, the work of plastic deformation, the size of plastic deformation zone, the amount of dissipated energy were used as a parameter determining the crack propagation rate [1-4].

The propagating crack interacts with the material which can be considered as a complex hierarchical structure. Mechanical behavior at different spatial levels can be described in terms of energy concepts. Monitoring of energy dissipation during fatigue crack propagation can give significant information about the kinetics of deformation processes and the current crack propagation rate [5-8].

Infrared thermography is an efficient method for estimating energy dissipation under mechanical testing. The main difficulty associated with application of this technique to the study of energy dissipation is related to the uncertainties in the solution of the inverse problem. The determination of energy dissipation under deformation can be obtained by the development of an additional system for direct monitoring of a heat flow. Such a system based on the Seebeck effect was developed at ICMM UB RAS [9].

In this work, we have derived an equation describing the evolution of plastic work at the crack tip. Following the idea given in [10], we have divided the plastic work and, as a consequence, energy dissipation at crack tip into two parts corresponding to reversible (cyclic) and monotonic plastic zones. Analysis of this approximation has shown the independence of energy dissipation in cyclic plastic zone from the crack growth. This dissipation is fully determined by the spatial size of a cyclic plastic zone and the characteristic diameter of the yield surface. For isotropic hardening materials, the change of the applied stress amplitude leads to the change of the characteristic diameter of the yield surface and, as consequence, to energy dissipation at a constant crack rate. Dissipation in the monotonic plastic zone is a function of both crack rate and characteristic diameter of the yield surface. This gives a well know correlation between fatigue crack rate and dissipated energy $[4,7]$.

To confirm the proposed approximation, we have compared it with the results of two fatigue crack propagation tests (uniaxial loading with constant stress intensity factor and biaxial loading with different biaxial coefficients). The experiments with constant stress intensity factor were reported in [11] for the first time. The main unexpected results of these experiments have shown that the energy dissipation measured by the contact heat flux sensor decreases during the crack propagation with the constant stress intensity factor. The proposed approximation demonstrates good qualitative agreement with the experimental data obtained during the uniaxial test.

The developed approach gives us an opportunity to generalize it for complex loading conditions. Analysis of the experimental data on crack propagation under biaxial loading has revealed similar qualitative features of energy dissipation [8]. We observed two stages of energy dissipation: constant value at the first stage and sharp increase at its final stage. The comparison of the phenomenological predictions and the obtained experimental results shows good qualitative agreement as well.

\section{EXPERIMENTAL SETUP}

wo types of experiments were carried out to analyze energy dissipation under fatigue crack propagation in metals. We conducted uniaxial tests at constant stress intensity factor and tests on biaxial loading with a constant stress amplitude. Fig. 1 shows the geometry of samples examined during the tests.

Samples for uniaxial testing were made of stainless steel AISE 304. The experiments were carried out at Bundeswehr University Munich. The samples were subjected to cyclic loading of $20 \mathrm{~Hz}$ at constant stress intensity factor and at loading ratio $\mathrm{R}=-1$. The crack length was measured by the potential drop method. The electrical potential drop method has been chosen due to its ability to monitor fatigue crack propagation and to set the stress intensity factor controlling a feedback. In this case, the potential method provides sensitivity up to $0.02 \mathrm{~mm}$ for a d.c. (direct current) of $5 \mathrm{~A}$.

The experimental program includes four tests with constant stress intensity factor. The constant stress intensity factors are $15 \mathrm{MPa} \mathrm{m}^{1 / 2}, 17.5 \mathrm{MPa} \mathrm{m}{ }^{1 / 2}, 20 \mathrm{MPa} \mathrm{m}{ }^{1 / 2}$ and $22.5 \mathrm{MPa} \mathrm{m}^{1 / 2}$, and the crack rates are $2.0076 \mathrm{e}-08 \mathrm{~m} / \mathrm{cycle}, 6.6391 \mathrm{e}-08$ $\mathrm{m} /$ cycle, $1.0245 \mathrm{e}-07 \mathrm{~m} /$ cycle, and $1.7177 \mathrm{e}-07 \mathrm{~m} /$ cycle, respectively. The first part of each experiment was performed subject to loading with a constant stress amplitude to initiate a $1 \mathrm{~mm}$ long fatigue crack, and the second part - at constant stress intensity factor until a $8 \mathrm{~mm}$ long crack was obtained.

A similar experimental program was realized at ICMM UB RAS. The detailed description of the mechanical properties, geometry of the samples and corresponding test conditions are given in [9]. The Russian steel analogous to that used to prepare samples of different geometries was tested for the loading ratio $\mathrm{R}=0$. The stress intensity factors were equal to 25 $\mathrm{MPa} \mathrm{m} 1 / 2$ and $30 \mathrm{MPa} \mathrm{m} 1 / 2$ (for crack rates of $1.4 \mathrm{e}-07 \mathrm{~m} /$ cycle, $1.65 \mathrm{e}-07 \mathrm{~m} /$ cycle, respectively). The results of both experimental programs are similar. In the future analysis, we are going to use the results obtained for the AISE 304 
samples through the recording of both crack propagation modes at constant stress amplitude and at constant stress intensity factor in each test.

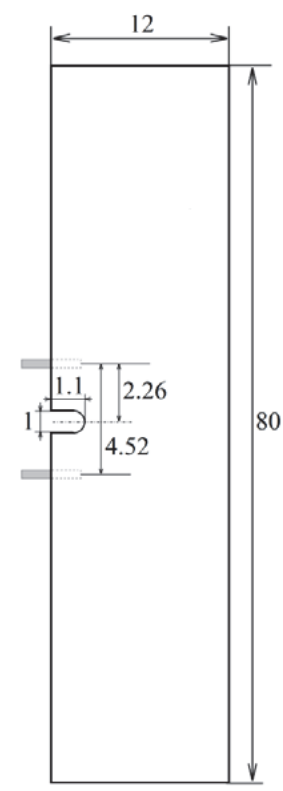

a)

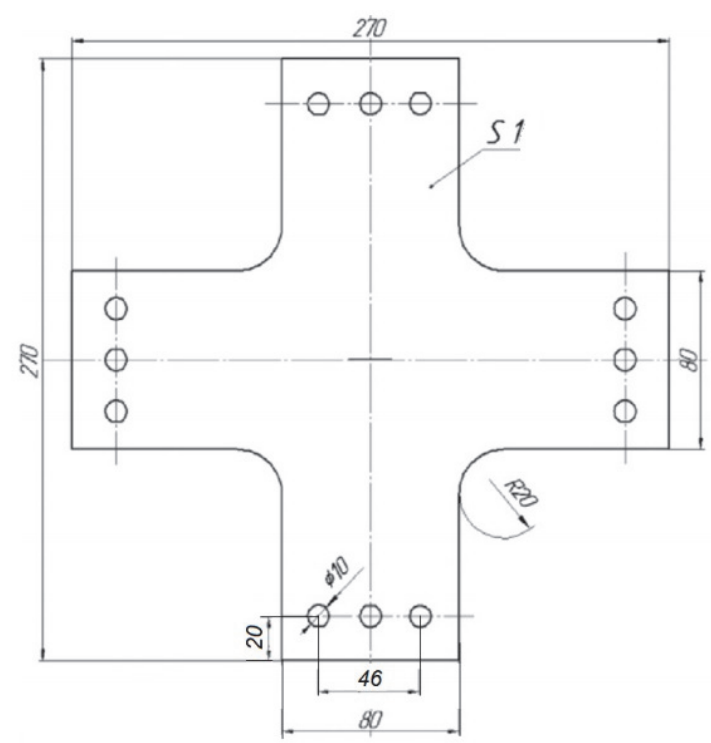

b)

Figure 1: Geometries of samples: for uniaxial test (a), for biaxial test (b).

Samples for biaxial testing were made of titanium alloy Grade 2 and were tested in the servo-hydraulic biaxial test system Biss BI-00-502 at Kazan Scientific Center of the Russian Academy of Sciences. While testing, the samples were subjected to cyclic loading of $10 \mathrm{~Hz}$ at constant stress amplitude and at different biaxial coefficients $\eta=\mathrm{Px} / \mathrm{Py}(1,0.7,0.5)$ and various ratios $\mathrm{R}(0.1,0.3,0.5)$. The crack length in the course of the experiment was measured by applying the optic method.

To analyze the dissipated energy at the crack tip, a contact heat flux sensor was used. The proposed sensor is based on the Seebeck effect, which is the reverse of the Peltier effect. The Peltier effect is a thermoelectric phenomenon, in which the passage of electric current through a conducting medium leads to the generation or absorption of heat at the point of contact (junction) of two dissimilar conductors. The quantity of heat and its sign depend on the type of materials in contact and on the electric current direction and strength. A thermal contact between the sample and the sensor is provided by the introduction of a thermal paste. These sensors were calibrated using a device reproducing the sample during the test with a controlled heat flux.

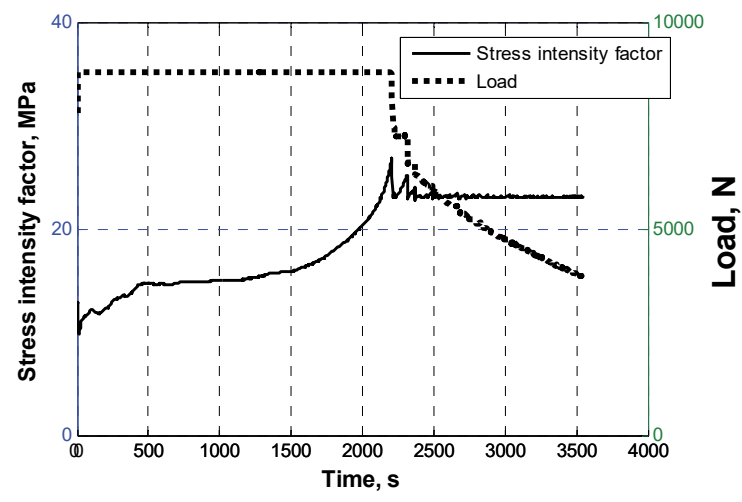

a)

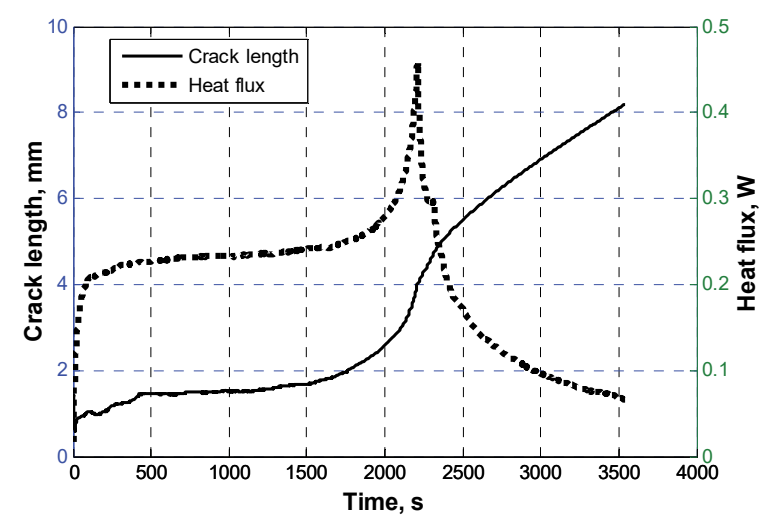

b)

Figure 2: Stress intensity factor/applied load (a) and energy dissipation/crack length (b) histories during the uniaxial fatigue test. 


\section{RESULTS OF FATIGUE EXPERIMENTS}

$\mathrm{T}$

he results of the uniaxial fatigue test are presented in Fig. 2. The test includes part with a constant stress amplitude up to the 2200-th second. We can observe a stable accelerated crack in the 500-th to 2200-th second interval accompanied by energy dissipation increase. From the 2200-th second, the stress intensity factor was kept constant. It leads to the decrease of the stress amplitude (fig.2a) and energy dissipation and results in nearly uniform crack propagation (fig.2b).

The results of the biaxial fatigue tests are presented in Fig. 3. Fig. 3a shows a plot of crack length versus time. Fig. 3b presents evidence that the fatigue crack propagates in the Paris regime.

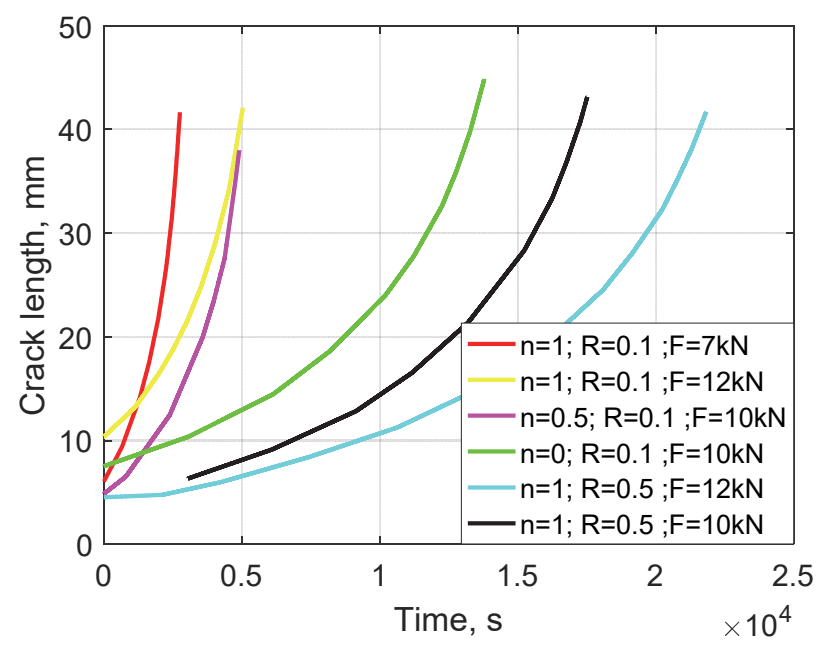

a)

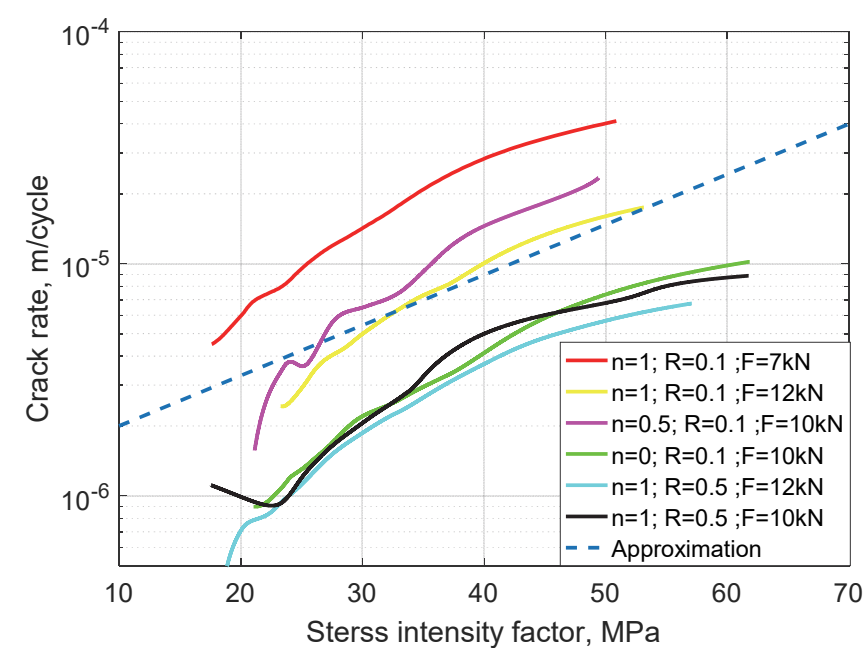

b)

Figure 3: Crack length versus time under biaxial loading conditions (different lines indicate different values of biaxial coefficients) (a), Paris curve (b) for biaxial test.

\section{THEORETICAL ANALYSIS OF HEAT DISSIPATION}

$\mathrm{F}$

ollowing the idea proposed in [10], we can start from a relationship between elastic and real deformation at the crack tip:

$$
\varepsilon_{\mathrm{ij}}^{e f}=\left(\frac{G}{G_{s}}\right)^{\frac{1}{2}} \varepsilon_{i j}^{e l},
$$

where $G$ - the shear modulus, $G$ s - secant shear modulus.

Eqn. (1) was originally proposed in [12] as a result of photo elastic experiment data treatment. Using the RambergOsgood relationship as the first approximation of mechanical behaviour of material under consideration $\gamma=\frac{\tau}{G}+A\left(\frac{\tau}{\tau_{0}}\right)^{n}$, we can write the following estimation for octahedral stress and link it with an elastic solution

$$
\tau_{\text {oct }}=\frac{\left(1+\frac{2(1+v)}{3} B \xi^{n-1}\right)^{\frac{1}{2}}}{1+B \xi^{n-1}} \tau_{\text {oct }}^{e l}
$$


where $A, \tau_{0}, n$ - material constants, $B=\frac{G A}{\tau_{0}}\left(\frac{\tau_{e}}{\tau_{0}}\right)^{n}, \xi=\frac{\tau_{o c t}}{\tau_{e}}, \tau_{e}$ - elastic limit.

The energy of plastic deformation in representative volume near the crack tip can be estimated as follows

$$
U_{p}=\frac{3}{2} \int \tau_{o c t} d \gamma_{o c t}=\frac{3}{2} \frac{A n}{n+1} \tau_{e}\left(\frac{\tau_{e}}{\tau_{0}}\right)^{n}\left(\frac{\tau_{o c t}}{\tau_{e}}\right)^{n+1}
$$

The energy increment caused by crack advance under monotonic loading can be written as

$$
d U_{p}=\frac{3}{2} A n \tau_{e}\left(\frac{\tau_{e}}{\tau_{0}}\right)^{n} \xi^{n} \frac{d \xi}{d l} d l
$$

where $l$ - crack length.

Using definition $\alpha=\frac{\tau_{o c t}^{e l}}{\tau_{e}}=\frac{K f_{e}^{\frac{1}{2}}}{3 \sqrt{r} \tau_{e}}=\left(\frac{r_{p} f_{e}}{r}\right)^{\frac{1}{2}}$ (here $K$ - stress intensity factor, $r_{p}-$ estimation for plastic zone size, $r$, $\theta$ - polar coordinates, $f_{e}$ - function of polar coordinate $\theta$, we can rewrite Eqn. (4) as

$$
d U_{p}=\frac{3}{2} A n \tau_{e}\left(\frac{\tau_{e}}{\tau_{0}}\right)^{n} \xi^{n} \frac{d \xi}{d \alpha} \frac{d \alpha}{d l} d l
$$

where

$$
\frac{d \alpha}{d l}=\frac{1}{2 \sqrt{r_{p} f_{e} r}}\left(f_{e} C+\frac{r_{p}}{r}\left(\sin \theta \frac{d f_{e}}{d \theta}+f_{e} \cos \theta\right)\right)
$$

To analyse plastic deformation at the crack tip under cyclic loading we need to divide energy dissipation in cyclic and monotonic plastic zones at the crack tip

$$
U_{p}^{t o t}=U_{p}^{g y c}+U_{p}^{m o n}
$$

The energy of representative volume at cyclic zone can be estimated as

$$
U_{p}^{g c}=\frac{3}{2} \tau_{e c} \gamma_{p c}
$$

where $\tau_{e c}$ - characteristic size of the yield surface, $\gamma_{p c}=\left(\tau_{o c t, c}-\tau_{e c}\right)\left(\frac{1}{G_{s}}-\frac{1}{G}\right)$ - amplitude of plastic deformation under an assumption of the validity of Ramberg-Osgood relationship ship, $\tau_{\text {oct }, c}$ - stress change in the representative volume. The full energy of cyclic plastic zone can be calculated as a double integral over the region (S) bounded on the outside of the monotonic plastic deformation zone and inside of the fracture zone

$$
U_{p}^{c y c}=2 \int_{s}^{\pi} \int_{0}^{\pi} \frac{3}{2} \tau_{e c}^{2}\left(\frac{1}{G_{s}}-\frac{1}{G}\right)\left(\frac{\tau_{o c t, c}}{\tau_{e c}}-1\right) r d r d \theta
$$

The simple approximation of plastic zone boundary can be given by $r=r_{p, c} f_{e}$, for cyclic-fracture zone boundary - 


$$
r=r_{p, c} f_{e} \frac{\tau_{e c}}{\tau_{f r}}
$$

The energy increment in cyclic plastic zone can be written as

$$
\frac{d U_{p}^{c y c}}{d N}=3 \tau_{e c}^{2}\left(\frac{1}{G_{s}}-\frac{1}{G}\right) \int_{S} \int_{0}^{\pi} \frac{d \tau_{o c t, c}}{d \tau_{o c t}^{e l}} \frac{d \tau_{o c t}^{e l}}{d N} r d r d \theta
$$

The integration of Eqn. (5) gives $\cdot \frac{d U_{p}^{c y c}}{d N}=0$.. It means that dissipation in cyclic plastic zone doesn't depend on the crack advance but it is fully determined by the applied load. The energy dissipation in monotonic plastic zone can be estimated as

$$
U_{p}^{m o n}=\frac{3}{2} \tau_{e} \gamma_{p}
$$

Energy increment per one cycle can be written as

$$
\frac{d U_{p}^{m o n}}{d N}=3 \tau_{e}\left(\frac{1}{G_{s}}-\frac{1}{G}\right) \int_{S_{1}} \int_{0}^{\pi} \tau_{o c t} \frac{d \tau_{o c t}}{d \tau_{o c t}^{e l}} \frac{d \tau_{o c t}^{e l}}{d l} \frac{d l}{d N} r d r d \theta
$$

Solution of (13) for the case of $\frac{d r_{p}}{d a} \rightarrow 0$ gives linear relationship between energy increment and crack rate. Finally, the Eqns. (11) and (13) allow us to propose the following approximation for energy of plastic deformation at the fatigue crack tip

$$
\frac{d \mathrm{U}_{p}^{\text {tot }}}{d \mathrm{~N}}=W_{1}\left(A_{\tau}^{2}, r_{p}\right)+W_{2}\left(A_{\tau}^{2}, r_{p}\right) \frac{d l}{d \mathrm{~N}}=a_{1} A_{\tau}^{2}+a_{2} A_{\tau}^{2} \frac{d l}{d N}
$$

where $A_{\tau}$-applied stress amplitude which determines the diameter of yield surface.

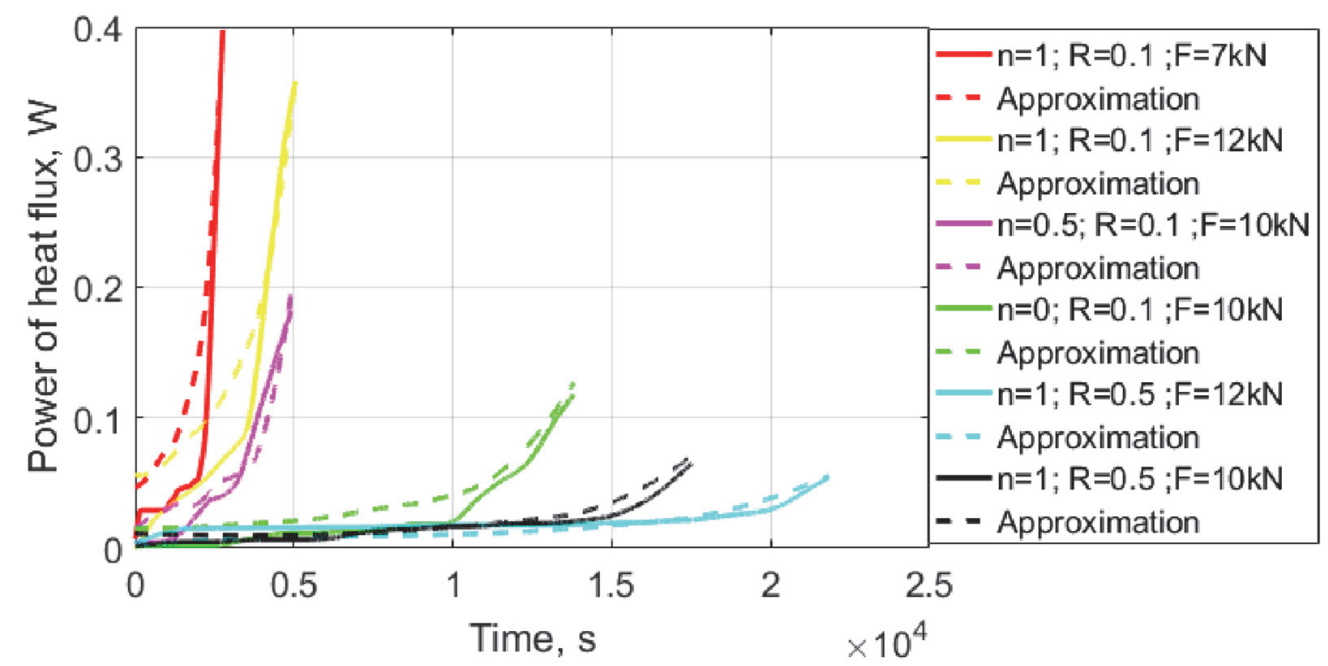

Figure 4: Energy dissipation histories during the biaxial test carried at constant stress amplitude (Solid line - experimental results, dotted line-approximation (14)). 


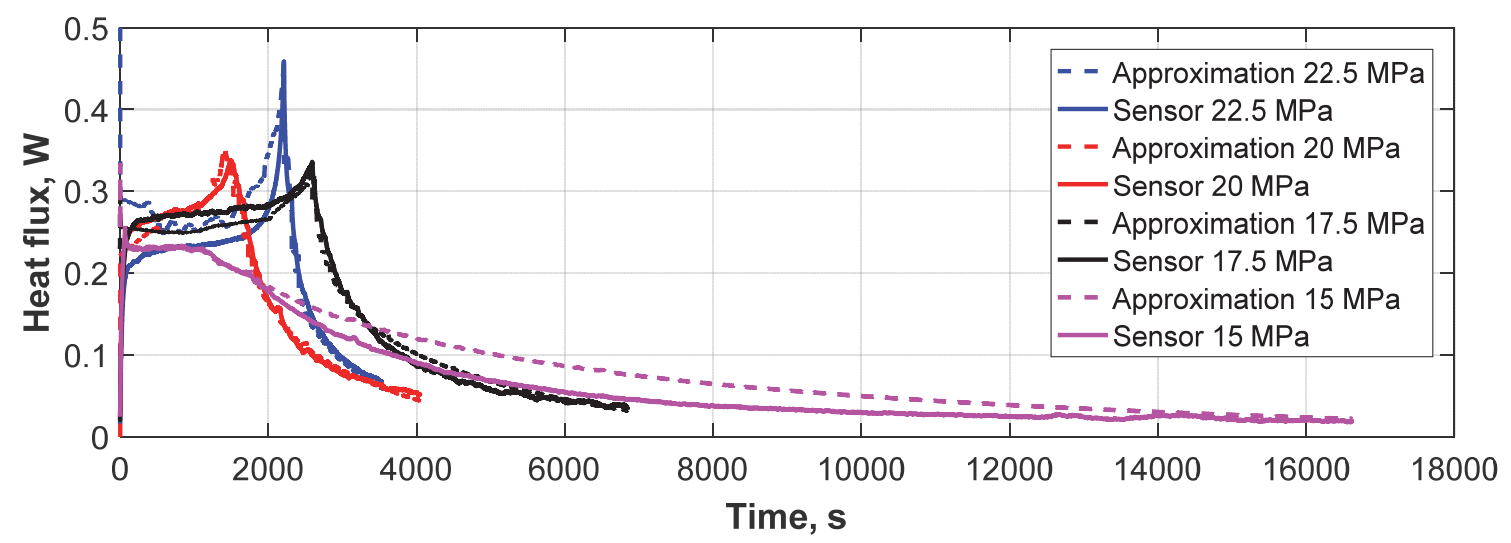

Figure 5: Energy dissipation histories during the uniaxial test carried out at constant stress amplitude and at constant stress intensity factor (Solid line - experimental results, dotted line -approximation (14)).

The process of heat dissipation is determined by the plastic work. This relationship could be complex due to the peculiarities of energy storage in material structure but taking into account the simplicity of Eqn. (13) we will use the linear dependence of heat dissipation versus energy of plastic deformation for the first approximation of experimental results.

The Eqns. (2)-(4) are used for the qualitative analysis of plastic deformation into one loading cycle. Qusistatic loading and unloading of materials near crack tip are considered to approximate dissipation caused one cycle. The analysis allows us to divide the dissipations caused by monotonous and revers loadings and propose both explanation and approximation for energy dissipation process. The qualitative calculation of energy dissipation based on this model will request more precise analysis and determination and correct exponent in Ramberg-Osgood model.

Figs. 4, 5 present the comparison of approximation (14) and results of the contact heat flux sensor. The Eqn. (14) gives a good qualitative description of peculiarities of heat dissipation in both regimes with the constant stress amplitude and constant stress intensity factor. In case of uniaxial loading for constant stress amplitude the plastic work and, as a consequence, energy dissipation at the crack tip is determined by crack rate as is shown in $[7,8]$ but for constant crack rate we can observe the regimes with the decrease of the heat dissipation caused by the decrease of the applied stress amplitude. For biaxial loading there is good agreement between experimental and theoretical results.

\section{CONCLUSIONS}

$\mathrm{I}$ $\mathrm{n}$ this work, experimental and theoretical studies of energy dissipated at fatigue crack tip in AISE 304 steel were carried out. Two types of experiments were performed: uniaxial loading tests and biaxial loading tests with constant stress amplitude for different values of the biaxial coefficient. For samples under uniaxial loading, two regimes of crack propagation were realized: constant stress amplitude and constant stress intensity factor. The results of the experiments have shown that at constant stress amplitude there is an increase in energy dissipation correlated with the fatigue crack rate. Crack propagation at constant stress intensity factor is accompanied by a decrease in energy dissipation. This effect was observed independently in two different experimental programs.

To provide an adequate explanation for the observed results, a theoretical analysis was performed in order to study the plastic work at the fatigue crack tip taking into account the evolution of both monotonic and cyclic plastic zones. This analysis allowed us to propose a simple approximation for energy dissipation at the fatigue crack tip. The obtained theoretical results give a good qualitative description of the specific features of energy dissipation in both regimes: loading with constant stress amplitude and loading with constant stress intensity factor. For constant stress amplitude, plastic work and, as a consequence, energy dissipation at the crack tip were determined by the crack rate, but for constant crack rate regime the scenario with a drop in energy dissipation takes place. In the case of biaxial loading there were two stages of energy dissipation: a constant heat flux accompanied by crack initiation and a sharp increase in energy dissipation associated with the occurrence of a large crack. The theoretical approach proposed for calculating energy dissipation correlates well with the experimental results. 


\section{ACKNOWLEDGEMENTS}

his work was supported by the Russian Foundation for Basic Research (Grant No. 16-51-48003).

\section{REFERENCES}

[1] Matvienko, Yu.G., Morozov, E M. (2004). Calculation of the energy J-integral for bodies with notches and cracks, International Journal of Fracture, 125, pp. 249-261.

[2] Rosakis, P., Rosakis, A.J., Ravichandran, G., Hodowany, J. (2000). A thermodynamic internal variable model for the partitional of plastic work into heat and stored energy in metals, J. Mech. Phys. Solids, 48, pp. 581-607.

[3] Oliferuk, W., Maj, M., Raniecki, B. (2004). Experimental analysis of energy storage rate components during tensile deformation of polycrystals, Materials Science and Engineering, 374, pp. 77-81.

[4] Izyumova, A., Plekhov, O. (2014). Calculation of the energy J-integral in plastic zone ahead of a crack tip by infrared scanning, FFEMS, 37, pp. 1330-1337.

[5] Meneghetti, G., Ricotta, M. (2016). Evaluating the heat energy dissipated in a small volume surrounding the tip of a fatigue crack, International Journal of Fatigue, 92(2), pp. 605-615.

[6] Risitano, A., Risitano, G. (2013). Cumulative damage evaluation in multiple cycle fatigue tests taking into account energy parameter, International Journal of Fatigue, 48, pp. 214-222.

[7] Ranganathan, N., Chalon, F., Meo, S. (2008). Some aspects of the energy based approach to fatigue crack propagation, International Journal of Fatigue, 30(10-11), pp. 1921-1929.

[8] Bär, U., Vshivkov, A., Plekhov, O. (2015). Combined lock-in thermography and heat flow measurements for analysing heat dissipation during fatigue crack propagation, Fracture and structural integrity, 34, pp. 521-530.

[9] Vshivkov, A., Iziumova, A., Bär, U., Plekhov, O. (2016). Experimental study of heat dissipation at the crack tip during fatigue crack propagation, Frattura ed Integrità Strutturale, 35, pp. 131-137.

[10] Raju, K. N. (1972). An energy balance criterion for crack growth under fatigue loading from considerations of energy of plastic deformation, International Journal of Fracture Mechanics, 8(1), pp. 1-14.

[11] Bär, U. (2016). Determination of dissipated energy in fatigue crack propagation experiments with lock-in thermography and heat flow measurement, Procedia Structural Integrity, 2, pp. 2105-2112.

[12] Dixon, J.R. (1965). Stress and strain distributions around cracks in sheet materials having various work-hardening characteristics, Ministry of Technology, National Engineering Laboratory, Materials Group: East Kilbride, Glasgow, Scotland, pp. 224-244. 